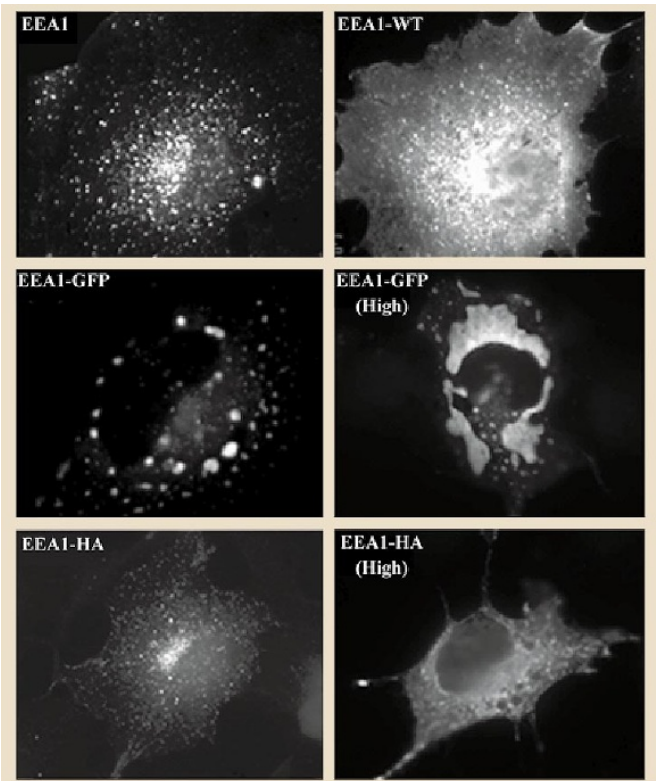

135 amino acids of EEA1 bound increasingly to liposomes containing increasing amounts of PtdIns(3)P (Fig. 1a). Chelation of zinc inhibited binding, indicating that the interaction between the fusion protein and PtdIns(3)P was through a RING zincfinger domain present in this carboxyterminal region (Fig. 1b). We saw no specific binding to liposomes containing other polyphosphoinositides (Fig. 1b).

A fusion protein containing GST and the pleckstrin-homology domain of GRP-1, which binds phosphatidylinositol-3,4,5trisphosophate with high affinity $\left(K_{\mathrm{d}}<1\right.$ $\mu \mathrm{M}$ ) (ref. 10), bound to liposomes containing phosphatidylinositol-3,4,5-trisphosphate, but not to liposomes containing PtdIns(3)P (Fig. 1b). Thus, the binding of the EEA1 RING finger to PtdIns(3)P is specific and of high affinity. Furthermore, the homologous domain in Hrs has been report$\mathrm{ed}^{11}$ to bind specifically to PtdIns(3)P. Thus this 'FYVE' domain seems to constitute a conserved binding motif for PtdIns(3)P.

A chimaera containing green fluorescence protein (GFP) at the carboxy terminus of EEA1 (EEA1-GFP) bound to liposomes containing $\operatorname{PtdIns(3)P,~but,~in~}$ contrast to wild-type EEA1, this chimaera also bound to liposomes composed of phosphatidylserine or phosphatidylserine and phosphatidylinositol (Fig. 1c).

When transfected into Cos-1 cells, EEA1-GFP caused the formation of lagoonlike structures (Fig. 2), which contained transferrin internalized from outside the cells (not shown), indicating that the structures may represent fused endosomes. Overexpression of wild-type EEA1 (Fig. 2, top), or of another EEA1 chimaera containing nine amino acids of the haemaglutinnin protein of influenza virus, did not produce this phenotype. Thus, the loss in binding specificity caused by GFP correlates with the formation of abnormal endosomal
Figure 2 EAA1-GPP prevents the correct formation of early endosomes. Cos-1 cells (control is shown at top left) were transfected with wild-type EEA1 (top right), EEA1-GFP (middle panels) or EEA1-haemagglutinin (bottom panels). Cells were stained with anti-EEA1 antiserum (top panels) or with anti-haemagglutinin antiserum (bottom panels). EEA1-GPP intrinsic fluorescence was visualized in unstained cells (middle panels). Examples of cells expressing relatively low (left panels) or high (right panels) levels of each construct are shown.

structures, indicating that the specific interaction between EEA1 and PtdIns(3)P may be vital in the control of endosome fusion. Varsha Patki, Deirdre C. Lawe, Silvia Corvera

Program in Molecular Medicine and Department of Cell Biology,

University of Massachusetts Medical School,

Worcester, Massachusetts 01605, USA

e-mail: silvia.corvera@ummed.edu

Joseph V. Virbasius, Anil Chawla

Biochemistry and Molecular Biology,

University of Massachusetts Medical School,

Worcester, Massachusetts 01605, USA

\footnotetext{
1. Patki, V. et al. Proc. Natl Acad. Sci. USA 94, 7326-7330 (1997).

2. Auger, K. R. et al. Cell 57, 167-175 (1989).

3. Stenmark, H. et al. J. Biol. Chem. 271, 24048-24054 (1996).

4. Weisman, L. S. \& Wickner, W. J. Biol. Chem. 267, 618-623 (1992).

5. Piper, R. C., Cooper, A. A, Yang, H. \& Stevens, T. H. J. Cell. Biol. 131, 603-617 (1995)

6. Komada, M., Masaki, R., Yamamoto, A. \& Kitamura, N. J. Biol. Chem. 272, 20538-20544 (1997).

7. Zheng, Y. et al. J. Biol. Chem. 271, 33169-33172 (1996).

8. Schu, P. V. et al. Science 260, 88-91 (1993).

9. Vanhaesebroeck, B. et al. Trends Biochem. Sci. 22, 267-272 (1997).

10. Klarlund, J. K. et al. Science 275, 1927-1930 (1997).

11. Gaullier, J.-M.et al. Nature 394, 432-433 (1998).
}

\section{Does practice shape the brain?}

Pantev et al. (Nature 392, 811-814; 1998) suggest that the degree of cortical reorganization and enhancement of the cortical response to musical notes depends on the age at which musicians first begin learning to play an instrument. Specifically, the younger the subjects were when they started to play, the larger was their cortical reorganization in recognition of piano tones. In addition to its biological interest, such a finding, if true, would have great implications for musical education. But we believe that the evidence presented by Pantev et al. is equally consistent with other interpretations.

Pantev et al. based their report on suggested correlations between the age at which musicians first began to play an instrument and the strength of cortical activation in response to piano tones when tested as adults. But the evidence for such correlations in their data is weak.

They present three correlations (for musicians with absolute pitch or with relative pitch, and for both groups combined). The (marginally significant) probability levels quoted are for one-tailed tests, used because 'negative correlations were predicted'. But it is possible that the cortical response to sensory experience might initially increase with age in young children, and so a positive correlation would be predicted. As both negative and positive correlations are possible, two-tailed tests should have been used in this case. Two-tailed tests show none of the reported correlations to be statistically significant at the traditional $5 \%$ level, and the confidence intervals for the correlation coefficients all include zero.

Even if more extensive experiments were to find a significant correlation, correlation does not indicate causation. The main contributors to the trends (if they exist) shown in Pantev et al.'s Fig. 2 are the musicians who began learning their instrument by 3-5 years of age. Musical tuition in such very young children is almost certainly associated with a high degree of parental musical skill or interest. At least two other explanations for the correlation would therefore be possible. First, perhaps only children with a particular type of cortical response to musical sounds are capable of learning an instrument from a very early age. Such a cortical response may have an inherited component. Second, it is possible that children brought up in musical families hear more music at a very young age, inducing more cortical reorganization. Neither explanation requires the children to have practised an instrument.

Pantev et al.'s comment that there was a "dependence of auditory [cortical]... representations on practice beginning before the age of about 10 years" ignores genotypic and environmental influences. It will be possible to establish the true nature of any relationship only by controlling for these effects. In the meantime, given our current state of knowledge, those wanting musical children might be well advised to examine carefully the musical abilities and compactdisc collections of potential mates, rather than investing in expensive music lessons for reluctant three-year-olds.

Pat Monaghan, Neil B. Metcalfe,

Graeme D. Ruxton

Institute of Biomedical and Life Sciences,

Graham Kerr Building, Glasgow University,

Glasgow G12 8QQ, UK 